\title{
Exercise Tolerance In Patients With Idiopathic Pulmonary Artery Hypertension: Insight Into Risk Thresholds And Prediction Capacity For 5-Year Mortality
}

\section{Zhihua Huang}

Fuwai Hospital, Chinese Academy of Medical Sciences and Peking Union Medical College

\section{Lu Yan}

Fuwai Hospital, Chinese Academy of Medical Sciences and Peking Union Medical College Zhihui Zhao

Fuwai Hospital, Chinese Academy of Medical Sciences and Peking Union Medical College Qing Zhao

Fuwai Hospital, Chinese Academy of Medical Sciences and Peking Union Medical College

\section{Yi Tang}

Hunan Normal University

Qi Jin

Fudan University

\section{Yi Zhang}

Fuwai Hospital, Chinese Academy of Medical Sciences and Peking Union Medical College

Xin Li

Fuwai Hospital, Chinese Academy of Medical Sciences and Peking Union Medical College

\section{Anqi Duan}

Fuwai Hospital, Chinese Academy of Medical Sciences and Peking Union Medical College

Meixi Hu

Fuwai Hospital, Chinese Academy of Medical Sciences and Peking Union Medical College

\section{Xiuping Ma}

Fuwai Hospital, Chinese Academy of Medical Sciences and Peking Union Medical College

\section{Qin Luo}

Fuwai Hospital, Chinese Academy of Medical Sciences and Peking Union Medical College Zhihong Liu ( $\nabla$ zhihongliufuwai@163.com)

Fuwai Hospital, Chinese Academy of Medical Sciences and Peking Union Medical College

\section{Research Article}


Keywords: Exercise tolerance, mortality, pulmonary hypertension, risk prediction, risk threshold

Posted Date: February 7th, 2022

DOI: https://doi.org/10.21203/rs.3.rs-1321407/v1

License: (c) (1) This work is licensed under a Creative Commons Attribution 4.0 International License. Read Full License 


\section{Abstract}

Background: Exercise tolerance is pivotal for risk-stratification in patients with idiopathic pulmonary artery hypertension (IPAH), yet optimal risk predictors and risk thresholds remain uncertain. This study aimed to investigate risk estimates of cardiopulmonary exercise testing (CPET) associated with 5-year mortality in patients with IPAH and explore their risk thresholds and prediction capacity.

Methods: Consecutive patients with IPAH who underwent right heart catheterization and CPET were retrospectively enrolled and followed up for five years. Multivariable Cox proportional hazards models were used to determine independent prognostic factors for mortality. The risk trend and threshold for mortality were exhibited using restricted cubic splines. Survival rates were estimated by Kaplan-Meier analysis stratified by various CPET parameters.

Results: Among 210 patients with IPAH (75.7\% female), 37 (17.6\%) died during a 34-month median follow-up. Three CPET variables were independently predictive of mortality in multivariable Cox regression analysis (all $P<0.05$ ), including oxygen uptake efficiency slope (OUES), peak oxygen pulse $\left(\mathrm{VO}_{2} / \mathrm{HR}\right)$, and peak oxygen consumption $\left(\mathrm{VO}_{2}\right)$, in descending order of prediction power $\left(x^{2}=37.39>\right.$ $35.96>35.57$ ). The levels of OUES at 0.91 , peak $\mathrm{VO}_{2} / \mathrm{HR}$ at $5.3 \mathrm{ml} \cdot \mathrm{min}^{-1} \cdot$ beat ${ }^{-1}$, and peak $\mathrm{VO}_{2}$ at 12.2 $\mathrm{ml} \cdot \mathrm{kg}^{-1} \cdot \mathrm{min}^{-1}$ were respectively identified as risk thresholds for mortality. Patients below these thresholds had significantly higher mortality risk (adjusted hazard ratio of OUES: 3.34; peak $\mathrm{VO}_{2} / \mathrm{HR}$ : 3.76; and peak $\mathrm{VO}_{2}: 1.56$ ) and lower survival rates (log-rank test, all $P<0.01$ ). The joint model (area under the curve [AUC] 0.838) of these CPET variables (AUC 0.724) and estimates in contemporary risk assessment tools (AUC 0.809 ) provided more excellent prediction capacity for 5-year mortality.

Conclusions: Suboptimal exercise tolerance indicated by OUES, peak $\mathrm{VO}_{2} / \mathrm{HR}$, and peak $\mathrm{VO}_{2}$ under certain thresholds posed a higher mortality risk in patients with IPAH, and their joint combination further improved the prediction capacity.

\section{Introduction}

Idiopathic pulmonary artery hypertension (IPAH) is a rare but devastating disease characterized by progressive remodeling of the small pulmonary arteries, leading to increased pulmonary vascular resistance, right heart failure (HF), and even death [1]. Despite advances in modern therapeutics for pulmonary artery hypertension (PAH), such as various combinations of drugs and modes of administration, the long-term prognosis remains unsatisfactory [2]. Exploring prognostic indicators of adverse outcomes is thereby warranted to facilitate a more accurate and urgent risk stratification.

Cardiopulmonary exercise testing (CPET) permits the evaluation of exercise intolerance [3]. It has become a valuable tool that has the potential of non-invasively estimating disease severity, therapeutic response, and prognosis in patients with pulmonary hypertension $(\mathrm{PH})$ [4-10]. Parameters that have been shown to embody these capabilities included peak oxygen consumption $\left(\mathrm{VO}_{2}\right)$, ventilation/carbon dioxide 
output slope (VE/ $/ \mathrm{VO}_{2}$ slope), end-tidal partial pressure of carbon dioxide at anaerobic threshold $\left(\mathrm{PETCO}{ }_{2} @ A T\right)$, and oxygen uptake efficiency slope (OUES). In recent years, there has been emerging data on the superiorities of OUES in predicting outcomes in patients with $\operatorname{HF}[11,12]$ and $\mathrm{PAH}[7,8]$ over other ventilatory efficiency parameters such as $\mathrm{VE} / \mathrm{VCO}_{2}$ slope. However, findings into CPET variables predictive of poor outcomes have been inconsistent and contradictory [4-10]. Notably, most of these variables and cut-off values were based on expert opinion or receiver operator characteristic curves (ROC). The dynamic risk range and thresholds for these CPET metrics associated with adverse outcomes, and the additional role in PAH risk-stratification according to current guidelines, were less explored.

Therefore, the purpose of this study was (1) to determine and compare the prognostic significance of risk parameters of CPET for predicting 5-year mortality, and (2) to explore their risk thresholds and whether they can further improve the prediction capacity compared to contemporary assessment tools for patients with IPAH.

\section{Methods}

\section{Participants}

Consecutive patients admitted to Fuwai Hospital from January 2015 to January 2020 with a newly diagnosed IPAH were retrospectively enrolled in this cohort. The diagnosis of IPAH was confirmed through right heart catheterization (RHC) according to the 2015 European Society of Cardiology (ESC)/European Respiratory Society (ERS) PH guidelines. Baseline clinical data including demographics, World Health Organization functional class (WHO-FC), 6-minute walk distance (6MWD), and key laboratory tests were collected upon admission. The study was approved by the Fuwai Hospital Ethics Committee (No. 2018-1100). Written informed consent was obtained from all enrolled participants.

\section{Cardiopulmonary Exercise Testing}

All recruited patients underwent CPET using the COSMED Quark CPET system before receiving specific therapy. The test was conducted by an experienced medical staff blinded to patients' medical records, and the equipment was calibrated before the individual test. Oxygen therapy was ceased for at least 30 minutes before performing CPET. After a 3-minute resting and a subsequent 3-minute warm-up of unloaded pedaling, patients started to exercise on a cycle ergometer with electromagnetic brake at a progressively incremental work rate of 5-30 $\mathrm{W} / \mathrm{min}$ according to the estimated exercise tolerance until reaching volitional exhaustion or symptoms limitation. Gas exchange parameters were measured by a metabolic cart on a breath-by-breath basis and averaged over 10-second intervals. Peak $\mathrm{VO}_{2}$ was defined as the highest obtained average oxygen consumption measured over 30 seconds in the last minute of exercise. Peak $\mathrm{VO}_{2}$ /heart rate $(\mathrm{HR})$ was calculated as peak $\mathrm{VO}_{2}$ divided by peak heart rate. The ventilatory AT was detected by combining the V-slope method and ventilatory equivalents [13]. The VE/ $/ \mathrm{VO}_{2}$ slope was identified as the slope of the linear regression relationship between minute ventilation (VE) and carbon dioxide production from resting to the peak exercise. The OUES represented the slope of the 
regression line between $\mathrm{VO}_{2}\left(y\right.$ axis, $\left.\mathrm{L} \cdot \mathrm{min}^{-1}\right)$ and the logarithmically transformed $\mathrm{VE}\left(x\right.$ axis, $\left.\mathrm{L} \cdot \mathrm{min}^{-1}\right)$ across the entire exercise course. Heart rate was measured every 1 minute. Heart rate reserve was calculated as the difference between peak and resting heart rate. Blood pressure was recorded at 3minute intervals and when the patients expressed peak exercise.

\section{Hemodynamic Studies}

Hemodynamic assessment with RHC was performed at resting to facilitate the diagnosis of IPAH. Parameters composed of mean pulmonary artery pressure (mPAP), pulmonary vascular resistance (PVR), pulmonary capillary wedge pressure (PCWP), right atrial pressure (RAP), mixed venous oxygen saturation, and cardiac output. Diagnostic criteria of IPAH include an mPAP $\geq 25 \mathrm{mmHg}$, and PVR $>3$ Wood units at rest in the presence of a normal PCWP $\leq 15 \mathrm{mmHg}$ when none of the definite PH etiologies (e.g., connective tissue disease, congenital heart defect, heritable, drugs or toxins-induced, severe lung or leftsided heart diseases, et al.) were found.

\section{Follow-up and Outcome Measures}

The primary clinical outcome was the rate of 5-year all-cause mortality. Follow-up information was obtained by telephone calls or routine clinical visits to monitor outcomes every three months during the first year and every six months after that. Patient deaths were confirmed by medical records or death certificates review. Overall survival time of patients was measured from the date of CPET until five years or to the date of patient death, whichever occurred first.

\section{Statistical Analysis}

Continuous and categorical variables were presented as the mean \pm standard deviations and counts (proportions), respectively. Comparisons between the survivors and non-survivors were made by twotailed independent samples $t$-tests for normally distributed variables and nonparametric Mann-Whitney $U$ test for not normally distributed variables, while chi-square test was used for categorical variables. Cox proportional hazards regression analysis was performed to evaluate the prognostic value of different parameters. Variables included in multivariable models were based on their clinical relevance and statistical significance in the univariable analysis. To identify the risk thresholds and trends, the continuous association of individual CPET estimates with mortality was modeled using restrictive cubic splines models with four knots at the $5^{\text {th }}, 35^{\text {th }}, 65^{\text {th }}$, and $95^{\text {th }}$ percentiles adjusting for gender (female), WHO-FC, PVR, and N-terminal prohormone of brain natriuretic peptide (NT-proBNP). The Kaplan-Meier survival curves were employed to depict the differences in the 5-year survival rate between patients with values above or below the risk thresholds estimated from cubic splines models. Curves were compared using the log-rank test. ROC analysis was used to evaluate the additional role of CPET parameters in risk prediction compared to European guidelines and identify the optimal prediction models. Correlations between CPET variables and pulmonary hemodynamics were also explored using the Pearson correlation coefficient. A two-sided $P<0.05$ was considered statistically significant. Statistical analyses were 
performed using $R$ statistical version 3.6.3 ( $R$ Project for Statistical Computing) within RStudio statistical software version 1.1.453.

\section{Results}

\section{Baseline Characteristics}

Of 211 consecutive patients with IPAH who underwent CPET, one was lost to follow-up due to lung transplantation, and the remaining 210 patients (51 men and 159 women, aged $31.9 \pm 10.0$ years) were included in the final analysis. The majority presented with WHO-FC II and III, consisting of 105 and 91 individuals. The mean levels of NT-proBNP were $1302.2 \mathrm{pg} / \mathrm{ml}$, with $6 \mathrm{MWD} 388.0 \pm 93.3$ meters and the cardiac index $2.8 \pm 0.9 \mathrm{ml} / \mathrm{min} / \mathrm{m}^{2}$. A total of 180 (85.7\%) patients received specific targeted therapy. Patients not receiving targeted therapy were mainly due to financial burden, intolerability, and cautions about adverse effects. During a median 34-month follow-up, 37 patients died in the entire cohort, accounting for a $17.6 \%$ mortality rate.

\section{Comparisons between Survivors and Non-Survivors}

Demographics did not differ between survivors and non-survivors, whereas non-survivors had significantly worse WHO-FC (III/IV, $64.9 \%$ vs. $42.8 \%, P=0.007)$, and decreased 6MWD (352.5 \pm 110.4 vs. $396.3 \pm 87.4, P=0.042$, Table 1). Non-survivors also had higher mPAP, PVR, RAP, and lower cardiac index (all $P<0.05$ ). No group differences were observed in CPET-derived parameters for peak work rate, peak respiratory exchange rate, and peak heart rate. Distinctively, non-survivors had lower levels of heart rate reserve, peak $\mathrm{VO}_{2}$, peak $\mathrm{VO}_{2} / \mathrm{HR}$, AT, peak systolic blood pressure (SBP), peak diastolic blood pressure, and had poorer ventilation efficiency (e.g., higher VE/VCO 2 Slope and lower PETCO ${ }_{2} @ A T$ ).

\section{Predictors of 5-Year Mortality}

In the univariable Cox regression analysis, WHO-FC, NT-proBNP, hemodynamic parameters, peak work rate, $\mathrm{HRR}$, peak $\mathrm{VO}_{2}$, peak $\mathrm{VO}_{2} / \mathrm{HR}$, peak SBP, AT, PETCO2@AT, VE $/ \mathrm{VCO}_{2}$ slope, and OUES were associated with an increased risk of 5-year mortality (all $P<0.05$, Table 2 ). To reduce collinearity between CPET parameters, separate multivariable Cox regression models were developed to assess their independent prognostic significance for mortality risk. As there were not enough primary outcomes, thereby avoiding overfitting for mortality, we only put gender (female), WHO-FC, PVR, and NT-proBNP into the multivariable analysis considering their clinical significance and chi-square values and $P$ values in the univariable analysis. Each line in Table 3 represents a separate model adjusting for gender (female), WHO-FC, PVR, and NT-proBNP. Among these CPET variables, OUES, peak $\mathrm{VO}_{2} / \mathrm{HR}$, and peak $\mathrm{VO}_{2}$ remained independently associated with mortality, in descending order of prediction power $\left(x^{2}=37.39>35.96>\right.$ 35.57). The lower these variables were, the higher the mortality risk (OUES: hazard ratio [HR] ( $95 \%$ confidence interval [CI]) 0.998 (0.996-0.999), $P=0.005$; peak VO 2 /HR: 0.717 (95\% Cl 0.534-0.964), $P=0.027$; and peak $\left.\mathrm{VO}_{2}: 0.868(95 \% \mathrm{Cl} 0.758-0.995), P=0.042\right)$. The distribution of their levels in different 
WHO-FC groups is demonstrated in Fig. 1. As cardiac function worsened, exercise capacity indicated by these parameters gradually declined $(P$-value for trend $<0.05)$.

\section{Risk Thresholds Exploration}

As shown in Fig. 2, optimal exercise tolerance reflected by higher OUES, peak $\mathrm{VO}_{2} / \mathrm{HR}$, and peak $\mathrm{VO}_{2}$ were beneficial in reducing mortality risk, yet associations of these metrics with mortality appeared to differ at low to moderate levels. For peak $\mathrm{VO}_{2}$, there was a positive linear association, with the lowest mortality risk seen in those with peak $\mathrm{VO}_{2}$ reaching approximately $20 \mathrm{ml} \cdot \mathrm{kg}^{-1} \cdot \mathrm{min}^{-1}$. The adjusted $\mathrm{HR}$ of 5year mortality appeared to continuously escalate as peak $V_{2}$ decreased below levels of $12.2 \mathrm{ml} \cdot \mathrm{kg}^{-1} \cdot \mathrm{min}^{-}$ 1 . In contrast, the relationship between OUES and mortality was curvilinear, with maximal mortality between 0.5 and 0.91 among which the risk reaches the peak at 0.72 , and with declining mortality risk above 0.91 . Peak $\mathrm{VO}_{2} / \mathrm{HR}$ between 1 and $5.3 \mathrm{ml} \cdot \mathrm{min}^{-1} \cdot$ beat $^{-1}$ is associated with the highest excess

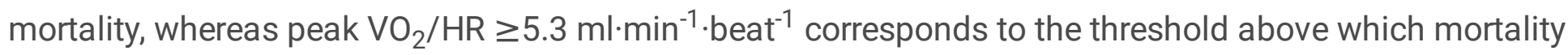
risk starts to decrease and reach a steady-state above the estimated level of $6 \mathrm{ml} \cdot \mathrm{min}^{-1} \cdot$ beat $^{-1}(P=0.828$ for non-linearity for OUES; $P=0.0324$ for non-linearity for peak $\mathrm{VO}_{2} / \mathrm{HR}$ ).

\section{Long-Term Survival Analysis}

Kaplan-Meier curves for survival stratified by OUES, peak $\mathrm{VO}_{2} / \mathrm{HR}$, and peak $\mathrm{VO}_{2}$ are shown in Fig. 3 . When these parameters were expressed dichotomously based on the risk threshold derived from restrictive cubic splines, OUES, peak $\mathrm{VO}_{2} / \mathrm{HR}$, and peak $\mathrm{VO}_{2}$ all predicted mortality well. Patients with OUES $<0.91(\mathrm{~N}=104)$ had a worse 5 -year survival rate than those with OUES $\geq 0.91(\mathrm{~N}=106)(72.1 \% \mathrm{vs}$. $92.5 \%, P=0.007)$. The unadjusted and adjusted $\mathrm{HR}$ of mortality was $4.84(95 \% \mathrm{Cl} 2.20-10.65)$ and 3.34 (95\% $\mathrm{Cl} 1.38-8.05)$, respectively. Patients with peak $\mathrm{VO}_{2} / \mathrm{HR}<5.3(\mathrm{~N}=106)$ had a worse 5-year survival rate than those with peak $\mathrm{VO}_{2} / \mathrm{HR} \geq 5.3 \mathrm{ml} \cdot \mathrm{kg}^{-1} \cdot \mathrm{min}^{-1}(\mathrm{~N}=104)(73.6 \%$ vs. $91.3 \%, P=0.002)$. The unadjusted $\mathrm{HR}$ of mortality was 3.42 (95\% $\mathrm{Cl} 1.61-7.24)$, and after adjustment for gender, WHO-FC, PVR, and NT-proBNP, the HR was $3.76(95 \% \mathrm{Cl} 1.61-8.82)$. Similarly, patients with peak $\mathrm{VO}_{2} \leq 12.2 \mathrm{ml} \cdot \mathrm{kg}^{-1} \cdot \mathrm{min}^{-1}$ exhibited a significantly lower survival rate than those with peak $\mathrm{VO}_{2}>12.2 \mathrm{ml} \cdot \mathrm{kg}^{-1} \cdot \mathrm{min}^{-1}(76.0 \% \mathrm{vs} .88 .7 \%$, $P=0.0045)$; and the adjusted $\mathrm{HR}$ for mortality was 1.56 (95\% $\mathrm{Cl} 1.32-5.44)$.

\section{Prediction Capacity for Mortality}

ROC curves are plotted to determine the overall accuracy for mortality risk prediction (Fig. 4). The combination of OUES, peak $\mathrm{VO}_{2} / \mathrm{HR}$, and peak $\mathrm{VO}_{2}$ (Model 4: area under the ROC curve [AUC] $=0.724,95 \%$ $\mathrm{Cl}$ 0.648-0.801) outperformed the models with their components (Model 1: OUES, AUC=0.714; Model 2: peak $\mathrm{VO}_{2} / \mathrm{HR}, \mathrm{AUC}=0.652$; and Model 3: peak $\mathrm{VO}_{2}$ : $\mathrm{AUC}=0.676$ ). Model 5 included simplified risk estimates i.e., WHO-FC, 6MWD, NT-proBNP and cardiac index according to European PH guidelines, and it showed moderate accuracy for risk prediction (AUC=0.809, 95\% $\mathrm{Cl} 0.697-0.922$ ). The joint combination (Model 6) of CPET parameters and the risk estimates in the simplified version of ESC/ERS PH guidelines 
was preferable to the remaining models given its largest AUC and most excellent accuracy for mortality risk prediction (Model 6: AUC=0.838, 95\% Cl 0.736-0.941; Specificity 89.9\%; Sensitivity $72.7 \%$ ).

\section{Correlations between CPET and Pulmonary Hemodynamics}

As the heat map showed (Fig. 5), blue represents positive correlation, whereas red represents negative correlation. The darker the color, the stronger is the correlation. The OUES was negatively correlated with PVR $(r=-0.49, P<0.001)$, $\operatorname{SPAP}(r=-0.43, P<0.001)$ and $\operatorname{mPAP}(r=-0.39, P<0.001)$, while negatively correlated with cardiac index $(r=0.42, P<0.001)$. The peak $\mathrm{VO}_{2} / \mathrm{HR}$ was negatively associated with PVR $(r=-0.46, P<0.001)$, SPAP $(r=-0.41, P<0.001)$, mPAP $(r=-0.38, P<0.001)$, while negatively correlated with cardiac index $(r=0.30, P<0.001)$. With respect to peak $\mathrm{VO}_{2}$, positive correlations with $\mathrm{PVR}$ $(r=-0.38, P<0.001)$, sPAP $(r=-0.35, P<0.001)$, mPAP $(r=-0.32, P<0.001)$, while negative correlations with cardiac index $(r=0.47, P<0.001)$ were observed. It seemed that PVR, cardiac index, and mPAP were potential indicators that mediate cardiopulmonary function in conferring mortality risk.

\section{Discussion}

In this long-term follow-up study of 210 patients with IPAH, the OUES, peak $\mathrm{VO}_{2} / \mathrm{HR}$, and peak $\mathrm{VO}_{2}$ were found to be independent markers for 5-year mortality. Notably, we offered a fresh perspective on the risk thresholds and prognostic impact of varying intensities of exercise capacity on mortality. The joint combination of these parameters can further improve the risk prediction capacity compared to contemporary assessment tools for patients with IPAH.

Identifying patients at higher mortality risk of IPAH remains an area of intense investigation and is of utmost importance to guide their therapeutic strategies. A growing body of evidence has shown the prognostic value of exercise tolerance in predicting survival in patients with $\mathrm{PAH}$. For example, peak $\mathrm{VO}_{2}$, PETCO ${ }_{2} @ A T, V E / V O_{2}$ slope, oxygen pulse, and peak SBP during CPET were correlated with survival in patients with PAH [14]. Nonetheless, only peak $\mathrm{VO}_{2}\left(\leq 10.4 \mathrm{~mL} \cdot \mathrm{kg}^{-1} \cdot \mathrm{min}^{-1}\right)$ and peak SBP $(\leq 120 \mathrm{mmHg})$ were independent predictors of mortality in the previous studies $[6,14]$. These findings were in part in agreement with the results of our study conducted in patients with IPAH. Remarkably, we further enhanced the understanding of the risk threshold $\left(12 \mathrm{ml} \cdot \mathrm{kg}^{-1} \cdot \mathrm{min}^{-1}\right)$ for capturing the dynamic trend in mortality risk. Consistently, exercise capacity is considered a vital component of the REVEAL 2.0 [15] and ESC/ERS risk stratification tools [16], and current guidelines recommend assessing exercise tolerance for decision-making $[1,17]$. Accumulating evidence suggests that exercise training confers a protective effect on exercise capacity as indicated by improvement of peak $\mathrm{VO}_{2}$ [18-20], possibly due to the increased capillary density of skeletal muscle [19] and optimized oxidative enzyme function in the peripheral muscles [21]. However, peak $\mathrm{VO}_{2}$ was not proved as an independent predictor in some other PAH cohort studies [6,22], probably ascribed to a lack of patient motivation and premature termination of exercise by the examiner. Overall, these discrepant findings could be attributed to the differences in sample size, 
associated conditions, or comorbidities. In this matter, our findings may be better applicable to IPAH with fewer comorbidities.

OUES, a useful submaximal exercise index of aerobic fitness, represents the absolute rate of increase in oxygen consumption per 10 -fold increase in ventilation. A higher OUES represents more oxygen being delivered in the body, while a lower OUES represents an enormous amount of ventilation required for given oxygen uptake. Three main factors can influence the values of OUES, including arterial carbon dioxide setpoint, carbon dioxide production, and death space ventilation [23, 24]. An earlier investigation showed that the arterial carbon dioxide setpoint did not vary between normal individuals and HF during exercise [25]. As such, both systemic perfusion (related to carbon dioxide production) and pulmonary perfusion (related to dead space ventilation) are related to the magnitude of OUES. Previous literature has shown that OUES was strongly correlated with peak $\mathrm{VO}_{2}$ and provided better predictive power over peak $\mathrm{VO}_{2}$ in patients with left-sided $\mathrm{HF}[11,26]$ and $\mathrm{PAH}[10]$. These observations were consistent with our findings regarding the superiority of OUES over peak $\mathrm{VO}_{2}$ in predicting adverse outcomes.

Peak $\mathrm{VO}_{2} / \mathrm{HR}$ reflects the amount of oxygen consumed per heartbeat (i.e., the product of stroke volume and arteriovenous oxygen difference during exercise). It indirectly indicates the maximal myocardial oxygen supply and cardiac function reserve under stress. The correlation analysis supports that peak $\mathrm{VO}_{2} / \mathrm{HR}$ is associated with decreased cardiac index and a higher mortality risk beyond the risk threshold. In comparison, a recent study explored the role of peak $\mathrm{VO}_{2} / \mathrm{HR}$ in patients with chronic obstructive pulmonary disease and concluded that it might be an indicative parameter of lung hyperinflation, $\mathrm{PH}$, and $\mathrm{HF}$ comorbidity [27]. Apart from this, we further supported the role of peak $\mathrm{VO}_{2} / \mathrm{HR}$ in estimating long-term mortality risk in patients with IPAH. It emphasizes maintaining an appropriate cardiovascular function by individualized exercise training and aggressive specific drug treatments.

Meanwhile, interestingly, we found that $\mathrm{VE} / \mathrm{VCO}_{2}$ slope did not predict the poor outcome, in contrast to the previous literature $[9,12,14,28]$, but consistent with others $[6,8,10,11]$. For example, Davies et al. [11] concluded that OUES rather than peak $\mathrm{VO}_{2}$ and $\mathrm{VE} / \mathrm{VCO}_{2}$ slope was the most powerful predictor in patients with left-sided HF. Similar findings were also observed in patients with PAH $[8,10]$. We proved that OUES provided better prognostic information than that provided by $\mathrm{VE} / \mathrm{VCO}_{2}$, and more meaningfully, shed light on their risk thresholds. This may be linked to the fact that OUES reflects not only the status of pulmonary perfusion but systemic perfusion. Nonetheless, opposite results showing that $\mathrm{VE} / \mathrm{VCO}_{2}$ plays a predominant role in predicting outcomes were observed in a study by Arena et al. [12]. Speculatively, underlying clues explaining these discrepancies include the $\beta$-blockers use, the study period, and the population inhomogeneity. Moreover, the 6MWD in our study did not associate with the outcome, which may be attributable to the selection of stable patients with relatively high values where peak $\mathrm{VO}_{2}$, peak $\mathrm{VO}_{2} / \mathrm{HR}$, and OUES become more sensitive to functional exercise capacity. 
The OUES seemed to be a vital predictive factor superior to the other CPET-derived parameters. There were curvilinear relationships between OUES and peak $\mathrm{VO}_{2} / \mathrm{HR}$ and mortality risk. The joint combination of independent indicators - OUES, peak $\mathrm{VO}_{2} / \mathrm{HR}$, and peak $\mathrm{VO}_{2}$ had a better prognostic prediction value than the simplified risk estimates in 5-year mortality. Close monitoring of these CPET variables may aid in better risk stratification and individualized therapy for patients with IPAH. The OUES and peak VO2/HR seem likely to further increase mortality risk at some low measurement levels. However, However, future studies are needed to explore the underlying exacerbating mechanisms and elaborate whether cardiopulmonary rehabilitation could serve as a critical strategy to alleviate exercise intolerance, ameliorate disease progression, and improve outcomes in patients with IPAH.

This long-term follow-up study characterized the continuous relationship between exercise tolerance and mortality risk. Greater statistical power using adjusted restrictive cubic splines models enhanced detection of the different associations seen with CPET risk thresholds and mortality, which have been rarely undertaken before. Several limitations also warrant discussion. First, our study only included patients with IPAH with a lower percentage of WHO-FC IV. The findings thereby may not be generalized to other PAH and those with poorer cardiac function. Second, although the data were prospectively collected, our analysis was retrospective in nature. Despite the multivariate adjustment, we cannot exclude the possibility of residual confounding and reverse causality. Finally, limited information was available on the PAH-specific treatments on the changes of the CPET variables. Future multi-center and large-scale studies are required to validate our findings and explore the potential aggressive therapeutic effects on exercise tolerance and disease outcomes.

\section{Conclusions}

Suboptimal exercise tolerance reflected by OUES, peak $\mathrm{VO}_{2} / \mathrm{HR}$, and peak $\mathrm{VO}_{2}$ under certain thresholds provides excellent prognostic information for 5-year mortality in patients with IPAH. The joint combination of these parameters and simplified risk estimates can improve the risk prediction capacity compared to contemporary assessment tools for patients with IPAH.

\section{List Of Abbreviations}

AT, anaerobic threshold

CPET, cardiopulmonary exercise testing

IPAH, idiopathic pulmonary artery hypertension

mPAP, mean pulmonary artery pressure

6MWD, 6-minute walk distance

NT-proBNP, N-terminal prohormone of brain natriuretic peptide 
OUES, oxygen uptake efficiency slope

PAH, pulmonary artery hypertension

PCWP, pulmonary capillary wedge pressure

$\mathrm{PETCO}_{2} @ \mathrm{AT}$, end-tidal partial pressure of carbon dioxide at anaerobic threshold

PVR, pulmonary vascular resistance

RAP, right atrial pressure

$\mathrm{RHC}$, right heart catheterization

VE, minute ventilation

$\mathrm{VE} / \mathrm{VCO}_{2}$ slope, ventilation/carbon dioxide

$\mathrm{VO}_{2}$, oxygen consumption

WHO-FC, World Health Organization functional class

\section{Declarations}

\section{Ethical approval and consent to participate}

Ethical approval for this study was obtained from the Institution Review Board of the Fuwai Hospital Ethics Committee (No. 2018-1100).

\section{Consent for publication}

Not applicable

\section{Availability of data and materials}

The datasets used and/or analyzed during the current study are available from the corresponding author on reasonable request.

\section{Competing interests}

The authors declare that they have no competing interests.

\section{Funding}

This study was funded by National Natural Science Foundation of China $(81370326,81641005$, and 81800056), Beijing Municipal Science and Technology Project (Z181100001718200), National Precision 
Medical Research Program of China (2016YFC0905602), Double First-Class Discipline Construction Fund of Peking Union Medical College and Chinese Academy of Medical Sciences (2019E-XK04-02), CAMS Innovation Fund for Medical Sciences (CIFMS) (2020-I2M-C\&T-B-055, 2021-I2M-C\&T-B-032), Capital's Funds for Health Improvement and Research (2020-2-4033), and Beijing Municipal Natural Science Foundation (7202168).

\section{Authors' contributions}

$\mathrm{ZH}$ and $\mathrm{LY}$ contributed to the study design, data analysis, manuscript drafting and revision. ZZ, QZ acquired data, critically reviewed, and revised the manuscript. YT, QJ, YZ, XL, AD, MH performed literature search. QL and ZL provided professional advice on data interpretation, critically reviewed, and revised the manuscript. All authors contributed substantially to the work and approved the final manuscript.

\section{Acknowledgements}

The authors would like to express their gratitude to all the participants for their cooperation and acknowledge funding agencies to support this work.

\section{References}

1. Galiè N, Humbert M, Vachiery JL, Gibbs S, Lang I, Torbicki A, et al. 2015 ESC/ERS Guidelines for the diagnosis and treatment of pulmonary hypertension: The Joint Task Force for the Diagnosis and Treatment of Pulmonary Hypertension of the European Society of Cardiology (ESC) and the European Respiratory Society (ERS): Endorsed by: Association for European Paediatric and Congenital Cardiology (AEPC), International Society for Heart and Lung Transplantation (ISHLT). Eur Heart J. 2016;37(1):67-119.

2. McLaughlin VV, Shah SJ, Souza R, Humbert M. Management of pulmonary arterial hypertension. J Am Coll Cardiol. 2015;65(18):1976-97.

3. Glaab T, Taube C. Practical guide to cardiopulmonary exercise testing in adults. Respir Res. 2022;23(1):9.

4. Pinkstaff SO, Burger CD, Daugherty J, Bond S, Arena R. Cardiopulmonary exercise testing in patients with pulmonary hypertension: clinical recommendations based on a review of the evidence. Expert Rev Respir Med. 2016;10(3):279-95.

5. Arena R, Lavie CJ, Milani RV, Myers J, Guazzi M. Cardiopulmonary exercise testing in patients with pulmonary arterial hypertension: an evidence-based review. J Heart Lung Transplant. 2010;29(2):159-73.

6. Wensel R, Francis DP, Meyer FJ, Opitz CF, Bruch L, Halank M, et al. Incremental prognostic value of cardiopulmonary exercise testing and resting haemodynamics in pulmonary arterial hypertension. Int $\mathrm{J}$ Cardiol. 2013;167(4):1193-8. 
7. Tang Y, Luo Q, Liu Z, Ma X, Zhao Z, Huang Z, et al. Oxygen Uptake Efficiency Slope Predicts Poor Outcome in Patients With Idiopathic Pulmonary Arterial Hypertension. J Am Heart Assoc. 2017;6(7).

8. Ramos RP, Ota-Arakaki JS, Alencar MC, Ferreira EV, Nery LE, Neder JA. Exercise oxygen uptake efficiency slope independently predicts poor outcome in pulmonary arterial hypertension. Eur Respir J. 2014;43(5):1510-2.

9. Deboeck G, Scoditti C, Huez S, Vachiéry JL, Lamotte M, Sharples L, et al. Exercise testing to predict outcome in idiopathic versus associated pulmonary arterial hypertension. Eur Respir J. 2012;40(6):14109.

10. Tang Y, Yao L, Liu Z, Xie W, Ma X, Luo Q, et al. Peak circulatory power is a strong prognostic factor in patients with idiopathic pulmonary arterial hypertension. Respir Med. 2018;135:29-34.

11. Davies LC, Wensel R, Georgiadou P, Cicoira M, Coats AJ, Piepoli MF, et al. Enhanced prognostic value from cardiopulmonary exercise testing in chronic heart failure by non-linear analysis: oxygen uptake efficiency slope. Eur Heart J. 2006;27(6):684-90.

12. Arena R, Myers J, Hsu L, Peberdy MA, Pinkstaff S, Bensimhon D, et al. The minute ventilation/carbon dioxide production slope is prognostically superior to the oxygen uptake efficiency slope. $\mathrm{J}$ Card Fail. 2007;13(6):462-9.

13. Mezzani A. Cardiopulmonary Exercise Testing: Basics of Methodology and Measurements. Ann Am Thorac Soc. 2017;14(Supplement_1):S3-s11.

14. Wensel R, Opitz CF, Anker SD, Winkler J, Höffken G, Kleber FX, et al. Assessment of survival in patients with primary pulmonary hypertension: importance of cardiopulmonary exercise testing. Circulation. 2002;106(3):319-24.

15. Benza RL, Gomberg-Maitland M, Elliott CG, Farber HW, Foreman AJ, Frost AE, et al. Predicting Survival in Patients With Pulmonary Arterial Hypertension: The REVEAL Risk Score Calculator 2.0 and Comparison With ESC/ERS-Based Risk Assessment Strategies. Chest. 2019;156(2):323-37.

16. Hoeper MM, Kramer T, Pan Z, Eichstaedt CA, Spiesshoefer J, Benjamin N, et al. Mortality in pulmonary arterial hypertension: prediction by the 2015 European pulmonary hypertension guidelines risk stratification model. Eur Respir J. 2017;50(2).

17. Grünig E, Eichstaedt C, Barberà JA, Benjamin N, Blanco I, Bossone E, et al. ERS statement on exercise training and rehabilitation in patients with severe chronic pulmonary hypertension. Eur Respir $\mathrm{J}$. 2019;53(2).

18. Grünig E, Lichtblau M, Ehlken N, Ghofrani HA, Reichenberger F, Staehler G, et al. Safety and efficacy of exercise training in various forms of pulmonary hypertension. Eur Respir J. 2012;40(1):84-92. 
19. Ehlken N, Lichtblau M, Klose H, Weidenhammer J, Fischer C, Nechwatal R, et al. Exercise training improves peak oxygen consumption and haemodynamics in patients with severe pulmonary arterial hypertension and inoperable chronic thrombo-embolic pulmonary hypertension: a prospective, randomized, controlled trial. Eur Heart J. 2016;37(1):35-44.

20. González-Saiz L, Fiuza-Luces C, Sanchis-Gomar F, Santos-Lozano A, Quezada-Loaiza CA, FloxCamacho $\mathrm{A}$, et al. Benefits of skeletal-muscle exercise training in pulmonary arterial hypertension: The WHOLEi+12 trial. Int J Cardiol. 2017;231:277-83.

21. Sabbahi A, Severin R, Ozemek C, Phillips SA, Arena R. The role of cardiopulmonary exercise testing and training in patients with pulmonary hypertension: making the case for this assessment and intervention to be considered a standard of care. Expert Rev Respir Med. 2020;14(3):317-27.

22. Ramos RP, Arakaki JS, Barbosa P, Treptow E, Valois FM, Ferreira EV, et al. Heart rate recovery in pulmonary arterial hypertension: relationship with exercise capacity and prognosis. Am Heart J. 2012;163(4):580-8.

23. Baba R, Nagashima M, Goto M, Nagano Y, Yokota M, Tauchi N, et al. Oxygen uptake efficiency slope: a new index of cardiorespiratory functional reserve derived from the relation between oxygen uptake and minute ventilation during incremental exercise. J Am Coll Cardiol. 1996;28(6):1567-72.

24. Hollenberg M, Tager IB. Oxygen uptake efficiency slope: an index of exercise performance and cardiopulmonary reserve requiring only submaximal exercise. J Am Coll Cardiol. 2000;36(1):194-201.

25. Sullivan MJ, Cobb FR. The anaerobic threshold in chronic heart failure. Relation to blood lactate, ventilatory basis, reproducibility, and response to exercise training. Circulation. 1990;81(1 Suppl):li47-58.

26. Lin YS, Huang HY, Lin WH, Wei J, Chen JC, Kuo LY, et al. Oxygen Uptake Efficiency Slope Predicts Major Cardiac Events in Patients With End-Stage Heart Failure. Transplant Proc. 2016;48(3):956-8.

27. Wu CW, Hsieh PC, Yang MC, Tzeng IS, Wu YK, Lan CC. Impact Of Peak Oxygen Pulse On Patients With Chronic Obstructive Pulmonary Disease. Int J Chron Obstruct Pulmon Dis. 2019;14:2543-51.

28. Groepenhoff H, Vonk-Noordegraaf A, van de Veerdonk MC, Boonstra A, Westerhof N, Bogaard HJ. Prognostic relevance of changes in exercise test variables in pulmonary arterial hypertension. PLoS One. 2013;8(9):e72013.

\section{Tables}

Table 1 Baseline Characteristics of Study Population. 
Variables

\begin{tabular}{l} 
Age, years \\
\hline Female, $\mathrm{n}(\%)$ \\
$\mathrm{BMI}, \mathrm{kg} / \mathrm{m}^{2}$ \\
\hline $\mathrm{WHO}-\mathrm{FC}, \mathrm{I} / \mathrm{II} / \mathrm{III} / \mathrm{IV}, \mathrm{n}$ \\
\hline $6 \mathrm{MWD}, \mathrm{m}$ \\
\hline NT-proBNP, pg/mL \\
\hline Targeted Therapy ${ }^{\dagger}, \mathrm{n}(\%)$ \\
\hline Hemodynamics
\end{tabular}

\begin{tabular}{|c|c|c|c|c|}
\hline RAP, mmHg & $4.9 \pm 4.1$ & $7.7 \pm 4.9$ & $5.4 \pm 4.4$ & $<0.001$ \\
\hline sPAP, mmHg & $88.5 \pm 24.2$ & $98.2 \pm 21.1$ & $90.2 \pm 23.9$ & 0.026 \\
\hline mPAP, mmHg & $56.8 \pm 16.0$ & $65.7 \pm 15.6$ & $58.4 \pm 16.3$ & 0.002 \\
\hline PVR, Wood Units & $12.0 \pm 5.8$ & $15.5 \pm 7.7$ & $12.6 \pm 6.3$ & 0.002 \\
\hline PCWP, mmHg & $7.2 \pm 3.4$ & $9.6 \pm 2.8$ & $7.6 \pm 3.4$ & $<0.001$ \\
\hline $\mathrm{CO}, \mathrm{ml} / \mathrm{min}$ & $4.7 \pm 1.6$ & $4.0 \pm 1.1$ & $4.6 \pm 1.5$ & 0.018 \\
\hline $\mathrm{Cl}, \mathrm{ml} / \mathrm{min} / \mathrm{m}^{2}$ & $2.9 \pm 0.9$ & $2.4 \pm 0.6$ & $2.8 \pm 0.9$ & 0.010 \\
\hline $\mathrm{SvO}_{2}, \%$ & $69.7 \pm 7.7$ & $68.2 \pm 7.4$ & $69.5 \pm 7.6$ & 0.285 \\
\hline \multicolumn{5}{|l|}{ CPET } \\
\hline Peak WR, W & $72.9 \pm 24.9$ & $64.4 \pm 25.3$ & $71.4 \pm 25.2$ & 0.062 \\
\hline Peak RER & $1.1 \pm 0.1$ & $1.2 \pm 0.3$ & $1.1 \pm 0.2$ & 0.404 \\
\hline Peak HR, $\min ^{-1}$ & $143.2 \pm 19.3$ & $137.9 \pm 24.8$ & $142.3 \pm 20.4$ & 0.225 \\
\hline HRR, $\min ^{-1}$ & $21.9 \pm 9.9$ & $17.2 \pm 10.3$ & $21.1 \pm 10.1$ & 0.009 \\
\hline Peak VO ${ }_{2}, \mathrm{ml} \cdot \mathrm{kg}^{-1} \cdot \mathrm{min}^{-1}$ & $13.2 \pm 3.6$ & $11.1 \pm 2.3$ & $12.8 \pm 3.5$ & $<0.001$ \\
\hline Peak $\mathrm{VO}_{2} / \mathrm{HR}, \mathrm{ml} \cdot \mathrm{min}^{-1} \cdot$ beat $^{-1}$ & $5.6 \pm 1.7$ & $4.9 \pm 1.5$ & $5.5 \pm 1.6$ & 0.023 \\
\hline Peak SBP, mmHg & $131.4 \pm 35.0$ & $111.5 \pm 19.1$ & $127.9 \pm 33.6$ & $<0.001$ \\
\hline Peak DBP, mmHg & $86.3 \pm 22.1$ & $78.7 \pm 13.0$ & $84.9 \pm 21.0$ & 0.007 \\
\hline AT, $\mathrm{ml} \cdot \mathrm{kg}^{-1} \cdot \mathrm{min}^{-1}$ & $10.0 \pm 2.5$ & $9.0 \pm 2.4$ & $9.8 \pm 2.5$ & 0.033 \\
\hline
\end{tabular}




\begin{tabular}{|c|c|c|c|c|}
\hline PETCO $_{2} @ A T, m m H g$ & $29.4 \pm 5.4$ & $26.1 \pm 5.0$ & $28.8 \pm 5.5$ & $<0.001$ \\
\hline VE/VCO ${ }_{2}$ Slope & $46.4 \pm 16.8$ & $57.8 \pm 18.5$ & $48.4 \pm 17.6$ & $<0.001$ \\
\hline OUES & $0.97 \pm 0.32$ & $0.76 \pm 0.83$ & $0.94 \pm 0.31$ & $<0.001$ \\
\hline
\end{tabular}

Data are expressed as mean \pm SD or number (percentage). AT, anaerobic threshold; BMI, body mass index; $\mathrm{Cl}$, cardiac index; $\mathrm{CO}$, cardiac output; DBP, diastolic blood pressure; HR, heart rate; HRR, heart rate reserve; mPAP, mean pulmonary artery pressure; 6MWD, 6-minute walk distance; NT-proBNP, N-terminal prohormone of brain natriuretic peptide; OUES, oxygen uptake efficiency slope; PCWP, pulmonary capillary wedge pressure; $\mathrm{PETCO}_{2} @ A T$, end-tidal partial pressure of carbon dioxide at anaerobic threshold; PVR, pulmonary vascular resistance; RAP, right atrial pressure; RER, respiratory exchange ratio; SBP, systolic blood pressure; SPAP, systolic pulmonary artery pressure; $\mathrm{SvO}_{2}$, venous oxygen saturation; $\mathrm{VCO}_{2}$, carbon dioxide output; $\mathrm{VE}$, minute ventilation; $\mathrm{VO}_{2}$, oxygen consumption; WHO-FC, World Health Organization Functional Class; WR, work rate.

Table 2 Univariate Cox Regression Analysis of Risk Factors Associated with Mortality. 


\begin{tabular}{lllll} 
Variables & Hazard Ratio & $95 \% \mathrm{Cl}$ & $x^{2}$ & $P$-value \\
\hline Age & 1.009 & $0.980-1.039$ & 0.372 & 0.546 \\
\hline Female & 0.548 & $0.279-1.077$ & 3.136 & 0.092 \\
\hline BMI & 0.945 & $0.859-1.041$ & 1.313 & 0.253 \\
\hline WHO-FC (III/IV vs I/II) & 2.188 & $1.112-4.308$ & 5.399 & 0.023 \\
\hline NT-proBNP & 1.001 & $1.000-1.001$ & 29.707 & $<0.001$ \\
\hline 6MWD & 0.997 & $0.993-1.002$ & 1.601 & 0.206 \\
\hline sPAP & 1.016 & $1.003-1.029$ & 6.121 & 0.014 \\
\hline mPAP & 1.025 & $1.007-1.043$ & 7.937 & 0.005 \\
\hline PCWP & 1.151 & $1.049-1.262$ & 9.15 & 0.003 \\
\hline PVR & 1.065 & $1.024-1.108$ & 10.032 & 0.004 \\
\hline RAP & 1.107 & $1.037-1.183$ & 9.524 & 0.002 \\
\hline CO $^{\text {PI }}$ & 0.707 & $0.534-0.936$ & 5.692 & 0.015 \\
\hline Cl $_{\text {SvO }}$ & 0.563 & $0.356-0.890$ & 5.988 & 0.014 \\
\hline Peak WR & 0.997 & $0.996-0.999$ & 15.737 & $<0.001$
\end{tabular}


AT, anaerobic threshold; BMI, body mass index; $\mathrm{Cl}$, cardiac index; $\mathrm{CO}$, cardiac output; DBP, diastolic blood pressure; HR, heart rate; HRR, heart rate reserve; mPAP, mean pulmonary artery pressure; 6MWD, 6-minute walk distance; NT-proBNP, N-terminal prohormone of brain natriuretic peptide; OUES, oxygen uptake efficiency slope; PCWP, pulmonary capillary wedge pressure; $\mathrm{PETCO}_{2} @ A T$, end-tidal partial pressure of carbon dioxide at anaerobic threshold; PVR, pulmonary vascular resistance; RAP, right atrial pressure; RER, respiratory exchange ratio; SBP, systolic blood pressure; SPAP, systolic pulmonary artery pressure; $\mathrm{SvO}_{2}$, venous oxygen saturation; $\mathrm{VCO}_{2}$, carbon dioxide output; $\mathrm{VE}$, minute ventilation; $\mathrm{VO}_{2}$, oxygen consumption; WHO-FC, World Health Organization; Functional Class; WR, work rate.

Table 3 Multivariable Cox Regression Analysis of CPET Variables Associated with Mortality.

\begin{tabular}{llllll} 
Variables & Hazard Ratio & $95 \% \mathrm{Cl}$ & $x^{2}$ & C-index & $P$-value \\
\hline VE$/ \mathrm{VCO}_{2}$ Slope $^{\dagger}$ & 1.009 & $0.992-1.025$ & 33.65 & 0.740 & 0.298 \\
\hline Peak SBP $^{\dagger}$ & 0.985 & $0.971-1.000$ & 34.84 & 0.765 & 0.056 \\
\hline $\mathrm{HRR}^{\dagger}$ & 0.978 & $0.942-1.016$ & 33.83 & 0.742 & 0.252 \\
\hline $\mathrm{AT}^{\dagger}$ & 0.963 & $0.835-1.111$ & 33.30 & 0.737 & 0.605 \\
\hline $\mathrm{PETCO}_{2} @ \mathrm{AT}^{\dagger}$ & 0.958 & $0.898-1.022$ & 33.61 & 0.741 & 0.195 \\
\hline Peak WR $^{\dagger}$ & 0.988 & $0.970-1.006$ & 34.21 & 0.744 & 0.180 \\
\hline Peak VO $_{2}{ }^{\dagger}$ & 0.868 & $0.758-0.995$ & 35.57 & 0.751 & 0.042 \\
\hline Peak VO $_{2} / \mathrm{HR}^{\dagger}$ & 0.717 & $0.534-0.964$ & 35.96 & 0.755 & 0.027 \\
\hline OUES $^{\dagger}$ & 0.998 & $0.996-0.999$ & 37.39 & 0.771 & 0.005
\end{tabular}

${ }^{\dagger}$ Each line in table represents a separate Cox regression model adjusting for Gender (female), WHO-FC, PVR, and NT-proBNP. AT, anaerobic threshold; $\mathrm{Cl}$, confidence interval; $\mathrm{HR}$, heart rate; HRR, heart rate reserve; NT-proBNP, N-terminal prohormone of brain natriuretic peptide; OUES, oxygen uptake efficiency slope; $\mathrm{PETCO}_{2} @ A T$, end-tidal partial pressure of carbon dioxide at anaerobic threshold; PVR, pulmonary vascular resistance; SBP, systolic blood pressure; $\mathrm{VCO}_{2}$, carbon dioxide output; $\mathrm{VE}$, minute ventilation; $\mathrm{VO}_{2}$, oxygen consumption; WHO-FC, World Health Organization Functional Class; WR, work rate.

\section{Figures}



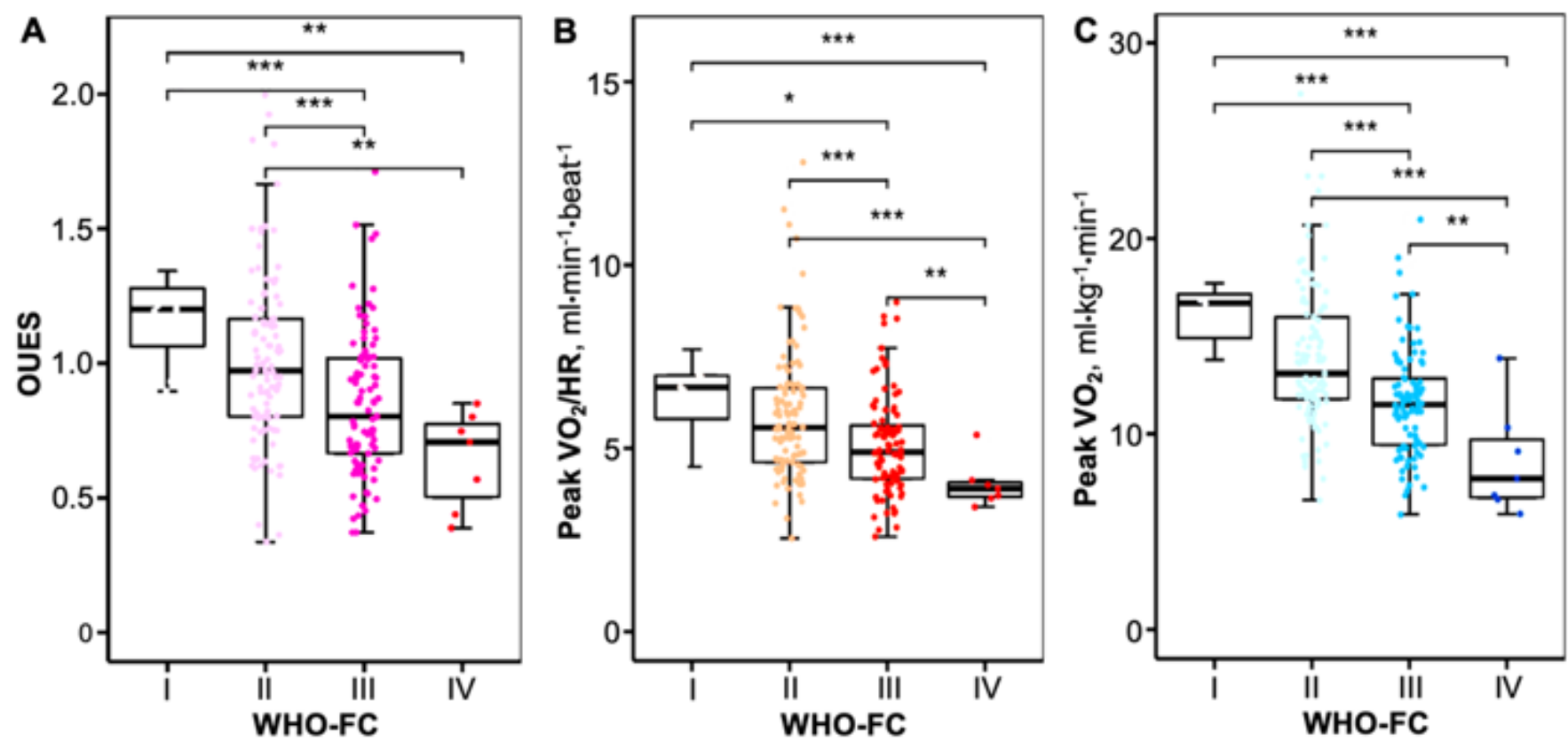

Figure 1

Boxplots of OUES (A), peak $\mathrm{VO}_{2} / \mathrm{HR}(\mathrm{B})$, and peak $\mathrm{VO}_{2}$ (C) in WHO-FC groups. OUES, oxygen uptake efficiency slope; $\mathrm{VO}_{2}$, oxygen consumption; $\mathrm{HR}$, heart rate; WHO-FC, World Health Organization Functional Class. Between-group differences: ${ }^{\star} P<0.05 ;{ }^{* \star} P<0.01 ;{ }^{* \star} P<0.001$.
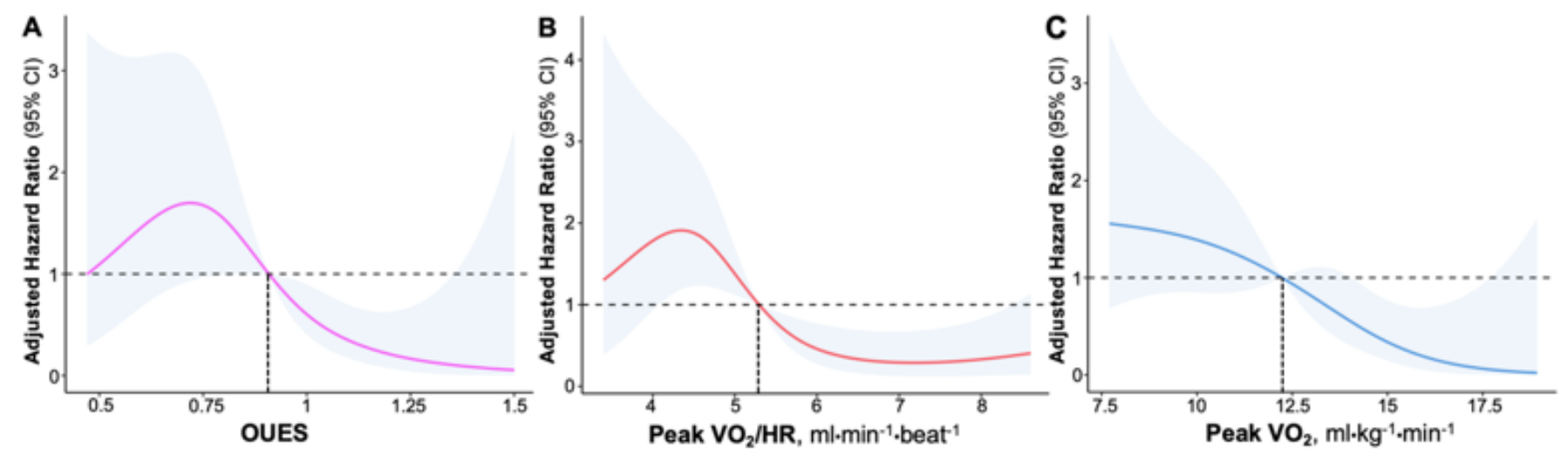

Figure 2

Restrictive cubic splines of associations between 5-year mortality risk and OUES (A), Peak $\mathrm{VO}_{2} / \mathrm{HR}$ (B), and Peak $\mathrm{VO}_{2}$ (C). The restrictive cubic splines models were adjusted for gender, WHO-FC, PVR, and NTproBNP, with knots at the $5^{\text {th }}, 35^{\text {th }}, 65^{\text {th }}$, and $95^{\text {th }}$ percentiles. The horizontal dash line indicates a null hazard ratio equal to 1 . The solid line denotes the estimated hazard ratio. The shaded area represents the $95 \%$ confidence limits. The hazard ratio is per each absolute increase of 1 unit of individual CPET 
parameter level. HR, heart rate; NT-proBNP, N-terminal prohormone of brain natriuretic peptide; OUES, oxygen uptake efficiency slope; PVR, pulmonary vascular resistance; $\mathrm{VO}_{2}$, oxygen consumption; WHO-FC, World Health Organization Functional Class.
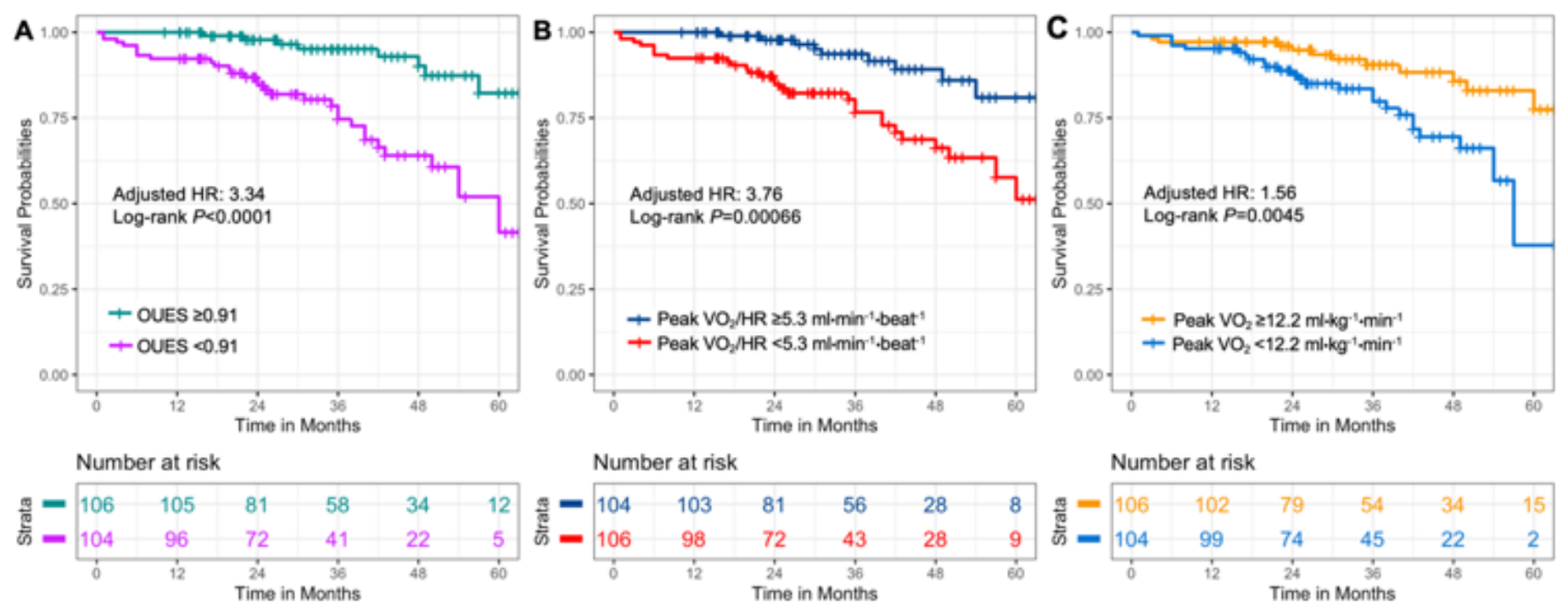

\section{Figure 3}

Kaplan-Meier curves for survival stratified by OUES (A), Peak VO $2 / \mathrm{HR}$ (B), Peak VO 2 (C). Adjusted HR indicates hazard ratio adjusted for gender, WHO-FC, PVR, and NT-proBNP. HR, heart rate; NT-proBNP, Nterminal prohormone of brain natriuretic peptide; OUES, oxygen uptake efficiency slope; PVR, pulmonary vascular resistance; $\mathrm{VO}_{2}$, oxygen consumption; WHO-FC, World Health Organization Functional Class. 


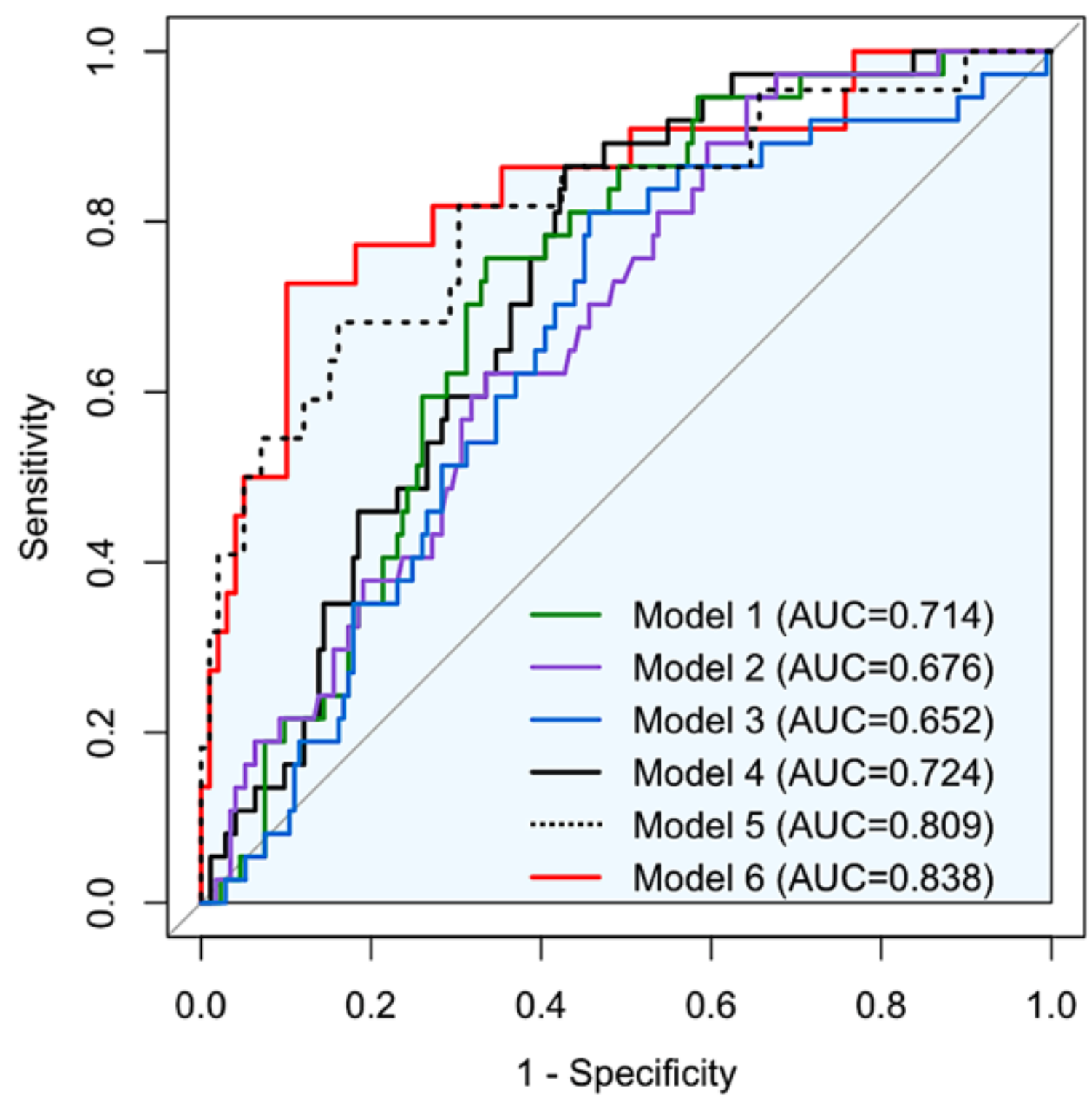

Figure 4

Risk prediction models for 5-year mortality in patients with IPAH. Each ROC curve represents one model. Parameters incorporated in each model are as follows. Model 1: OUES (AUC=0.714, 95\% $\mathrm{Cl} 0.635-0.793$ ). Model 2: peak $\mathrm{VO}_{2} / \mathrm{HR}(\mathrm{AUC}=0.652,95 \% \mathrm{Cl} 0.561-0.744)$. Model 3: peak $\mathrm{VO}_{2}(\mathrm{AUC}=0.676,95 \% \mathrm{Cl} 0.591-$ 0.762). Model 4: the combination of OUES, peak $\mathrm{VO}_{2} / \mathrm{HR}$ and peak $\mathrm{VO}_{2}(\mathrm{AUC}=0.724,95 \% \mathrm{Cl} 0.648-0.801)$. Model 5: risk estimates in the simplified version of the risk assessment strategy proposed by European $\mathrm{PH}$ guidelines, including WHO-FC, 6MWD, NT-proBNP, and Cardiac index (AUC=0.809, 95\% $\mathrm{Cl} 0.697-0.922$ ). Model 6: parameters in model 5 and model 6 (AUC=0.838 95\% Cl 0.736-0.941; Specificity 89.9\%; Sensitivity $72.7 \%$ ). AUC, area under the receiver operating characteristic curve; $\mathrm{Cl}$, confidence interval; $\mathrm{HR}$, heart rate; IPAH, idiopathic pulmonary artery hypertension; OUES, oxygen uptake efficiency slope; $\mathrm{PH}$, pulmonary hypertension; $\mathrm{ROC}$, receiver operator characteristic curve; $\mathrm{VO}_{2}$, oxygen consumption; $\mathrm{WHO}-\mathrm{FC}$, World Health Organization Functional Class. 


\begin{tabular}{|c|c|c|c|c|c|c|c|}
\hline VE $/ \mathrm{VCO}_{2}$ Slope & $* \star \star *$ & $\star \star \star \star *$ & $\star \star \star \star)$ & $* \star \star$ & * & $\star \star \star *$ & $\star \star \star *$ \\
\hline Peak SBP & 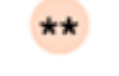 & 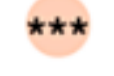 & $\star \star \star$ & & & & * \\
\hline HRR & 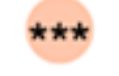 & 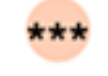 & $\star \star \star \star *$ & $\star \star \star \star$ & & $\star \star \star \star \Delta$ & $\star \star \star *$ \\
\hline AT & 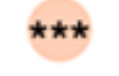 & ** & $\star \star$ & $\star \star \star$ & & * & $\star \star \star *$ \\
\hline PETCO $_{2} @ A T$ & $\star \star \star$ & $\star \star \star *$ & $\star \star \star *$ & $* \star \star *$ & * & $\star \star \star *$ & $\star \star * *$ \\
\hline Peak WR & $\star \star \star \star$ & $\star \star \star \star$ & $\star \star \star \star$ & & & $\star \star \star \star$ & $\star \star \star \star$ \\
\hline Peak VO 2 & $* \star \star *)$ & $\star \star \star *$ & $\star \star \star *$ & $* \star \star *$ & & $\star \star \star *$ & $\star * * *$ \\
\hline Peak $\mathrm{VO}_{2} / \mathrm{HR}$ & $\star \star \star \star$ & $\star \star \star \star$ & $\star \star \star \star ~$ & $\star \star$ & & $\star \star \star \star$ & $\star \star \star$ \\
\hline OUES & $\star \star \star *)$ & $\star \star \star *$ & $\star \star \star * *$ & 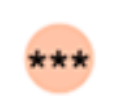 & & $\star \star \star \star *$ & $\star \star \star *$ \\
\hline
\end{tabular}

Figure 5

The heatmap of correlations between pulmonary hemodynamics and cardiopulmonary exercise testing parameters. Blue represents positive correlation, whereas red represents negative correlation. The darker the color, the stronger is the correlation. Asterisk reflects significant Pearson correlation coefficient: ${ }^{*} P<$ $0.05 ;{ }^{*} P<0.05 ;^{*} P<0.001$. 